\title{
Validation of XRD Stress Analyses Combining in-situ Tests and Integrated Peak Processing
}

\author{
B. Voillot ${ }^{1-2, a *}$, R. Billardon ${ }^{2, c}$, J.L. Lebrun ${ }^{3}$, F. Hild ${ }^{1, b}$ \\ ${ }^{1}$ LMT-Cachan, ENS Cachan/CNRS/Université Paris-Saclay, 61 avenue du Président Wilson \\ 94235 Cachan, France \\ ${ }^{2}$ Safran Landing Systems, Safran group, Inovel Parc Sud, 7 rue Général Valérie André \\ 78140 Velizy-Villacoublay, France \\ ${ }^{3}$ Université Paris-Est, Institut de Recherche en Constructibilité, ESTP, 94230 Cachan, France \\ avoillot@Imt.ens-cachan.fr, bhild@Imt.ens-cachan.fr, 'rene.billardon@safrangroup.com
}

Keywords: X-ray Diffraction, Ti5553 Alloy, in-situ Tensile Test, Peak Correlation, Integrated Methods

\begin{abstract}
The surface integrity has a significant effect on the fatigue life of structural components (e.g., landing gears). To estimate sub-surface residual stresses, X-ray diffraction (XRD) is applied to a Ti5553 alloy sample in an in-situ tensile test. This material is challenging since it is made of $\alpha$ and $\beta$ phases of different proportions, shapes and scales ranging from submicrometre to millimetre sizes. Therefore stress variations occur between grains. For millimetric probed volumes, the studied microstructure leads to shallow and noisy diffraction signals. It is shown that combing a new integrated method of diffraction peak registration and in-situ XRD measurements in tensile tests allows qualitative and quantitative results of residual stress analyses to be obtained for Ti5553 alloy samples.
\end{abstract}

\section{Introduction}

Aircraft landing systems are not only subjected to high dynamic load levels upon landing, but also to fatigue cycles during landing, take-off and taxiing. The structural parts of landing systems are made of alloys with high specific strength. The continuous development of such materials recently led to the introduction of forged and machined parts made of titanium alloys with high ultimate tensile strength [1]. The fatigue life of structures depends on intrinsic (i.e., bulk) and extrinsic (i.e., surface) properties [2,3]. Consequently, chemical and mechanical treatments are performed as part of the manufacturing process to enhance the resistance to corrosion and fatigue of all structural components (in particular parts made of high strength titanium alloys). Compressive residual stresses are created on the sub-surface of forged and machined parts. These residual stresses have to be evaluated to quantify their influence on the fatigue properties of structures. The most common non-destructive evaluation technique used to determine residual stresses is X-ray diffraction (XRD).

The aim of this work is to prove the feasibility of XRD for challenging stress analyses. Qualitative, quantitative and reliable estimation of stresses in coupons made of high strength twophase titanium alloy (i.e., Ti5553) are needed. In-situ tensile tests are carried out to compare stress estimations obtained via XRD measurements with other techniques (e.g., applied stress data using load cells). To post-process XRD measurements two in-house codes have been developed. The images shot during the experiment have been used to check that the probed volume has not moved during the experiment thanks to digital image correlation (DIC [4]).

\section{XRD stress analysis on two-phase titanium alloy}

One of the challenges of XRD analyses of two-phase alloys such as Ti5553 is the microstructure complexity [5,6], see Figure 1. Ti5553 is a metastable $\beta$-alloy made of body-centred cubic (BCC) 
matrix and hexagonal close-packed (HCP) inclusions (i.e., $\alpha$-phase) with different volume fractions, shapes and scales of grains ranging from sub-micrometre (i.e., lamellae) to millimetre size (i.e., $\beta$ grains). These dimensions are to be compared to the 1-mm in diameter X-ray spot that is used herein. The probed volume by the X-ray beam spreads over a few $\mathrm{mm}^{2}$ and penetrates down to $3 \mu \mathrm{m}$ under the surface. The consequence is the heterogeneity of the material concerned with XRD measurements, especially the variability in number and shape of $\alpha$ - and $\beta$-grains within the probed volume. These grains may experience small variations in lattice parameters or local stresses, which result in small strain variations. Consequently, XRD peaks are shallow, which make XRD analyses challenging. In the present work, only stresses in the $\alpha$-phase will be estimated. The use of $\{213\}$ planes corresponds to Bragg's angle $2 \theta=140.6^{\circ}$. The elastic parameters chosen for the present analyses are linked to the $\alpha$-phase for an isotropic elastic behaviour in a two-phase titanium alloy [7] (i.e., $\frac{1}{2} S_{2}=\frac{(1+v)}{E}=11.9 \times 10^{-6} \mathrm{MPa}^{-1}$, where $v$ and $E$ are respectively Poisson's ratio and Young's modulus for the $\alpha$-phase).
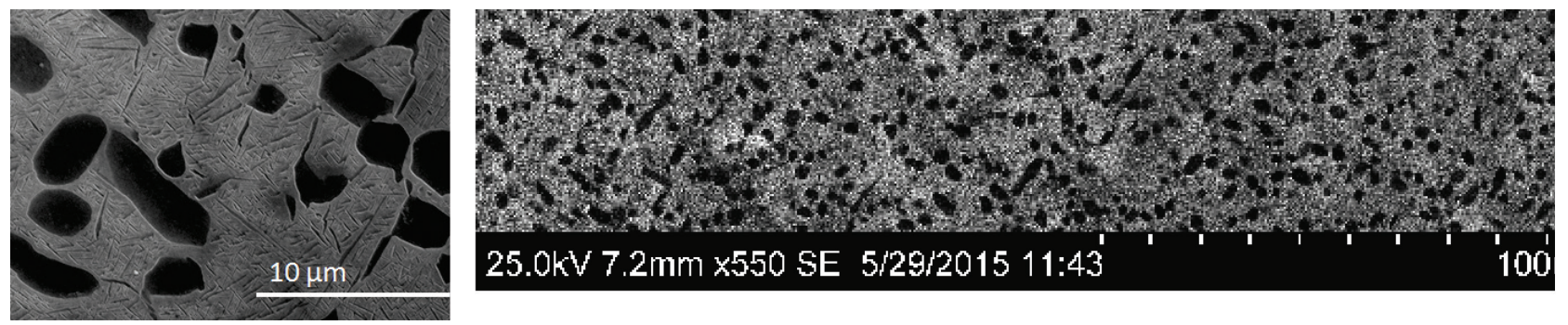

Figure 1: SEM pictures of Ti5553 alloy from a bogie of a landing gear. Sub-micrometre size $\alpha$ lamellae (left) and micrometre size $\alpha$-nodules (right) embedded in $\beta$-matrix

When a material is loaded (as herein) or exhibits residual stresses (e.g., landing gears after shotpeening), the crystal lattices deform. XRD enables variations of interreticular distances to be measured by analysing peaks due to diffraction phenomena in crystalline materials. The link between diffraction peak angle and interreticular distances is given by Bragg's law. Elastic stresses are then evaluated by estimating peak shifts with the chosen elastic law. Post-processing is carried out with the so-called $\phi \psi$ isotropic elastic law [8].

Morphological variations in two-phase materials induce different stresses within grains. Therefore the stresses differ within the considered volume, thereby inducing micro-shifts of diffraction peaks in the diffractogram. For millimetre-size probed volumes, such microstructures create shallow and noisy diffraction peaks. The post-processing steps need special care to be exercised [8,9]. Results of stress evaluations in such two-phase alloys thus need to be validated with independent measurements. It is proposed to use an in-situ tensile test for which the applied global forces are measured by the load cells and can be linked to stresses measured via XRD. In the present case, the stress states may slightly vary between two grains of the same coupon [5,6]. This is an additional issue for the present work since locations of XRD measurements are not perfectly reproducible and some uncertainties are expected.

\section{In-situ tensile test for XRD stress analyses}

A peak correlation method for XRD measurements is applied and compared with a new approach that is based on integrated algorithms [10], which are used in DIC techniques [4]. The in-situ XRD measurements are carried out in the centre of the top face of a dogbone flat coupon made of Ti5553 alloy (Figure 2). After electropolishing, a speckle pattern is sprayed on the top surface to enable images to be registered via DIC. Two opposite actuators are used so that the monitored zone is motionless (this hypothesis was checked with DIC). 


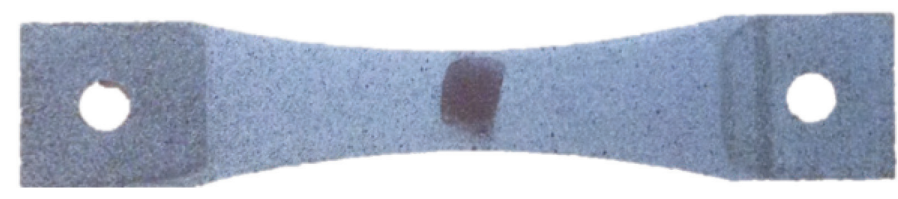

Figure 2: Ti5553 speckled coupon with an unpainted area to enable for XRD measurement. Sample dimensions: $50 \times 6 \times 0.5 \mathrm{~mm}^{3}$

Figure 3 illustrates the in-situ setup consisting of the miniature testing machine within the XRD goniometer.

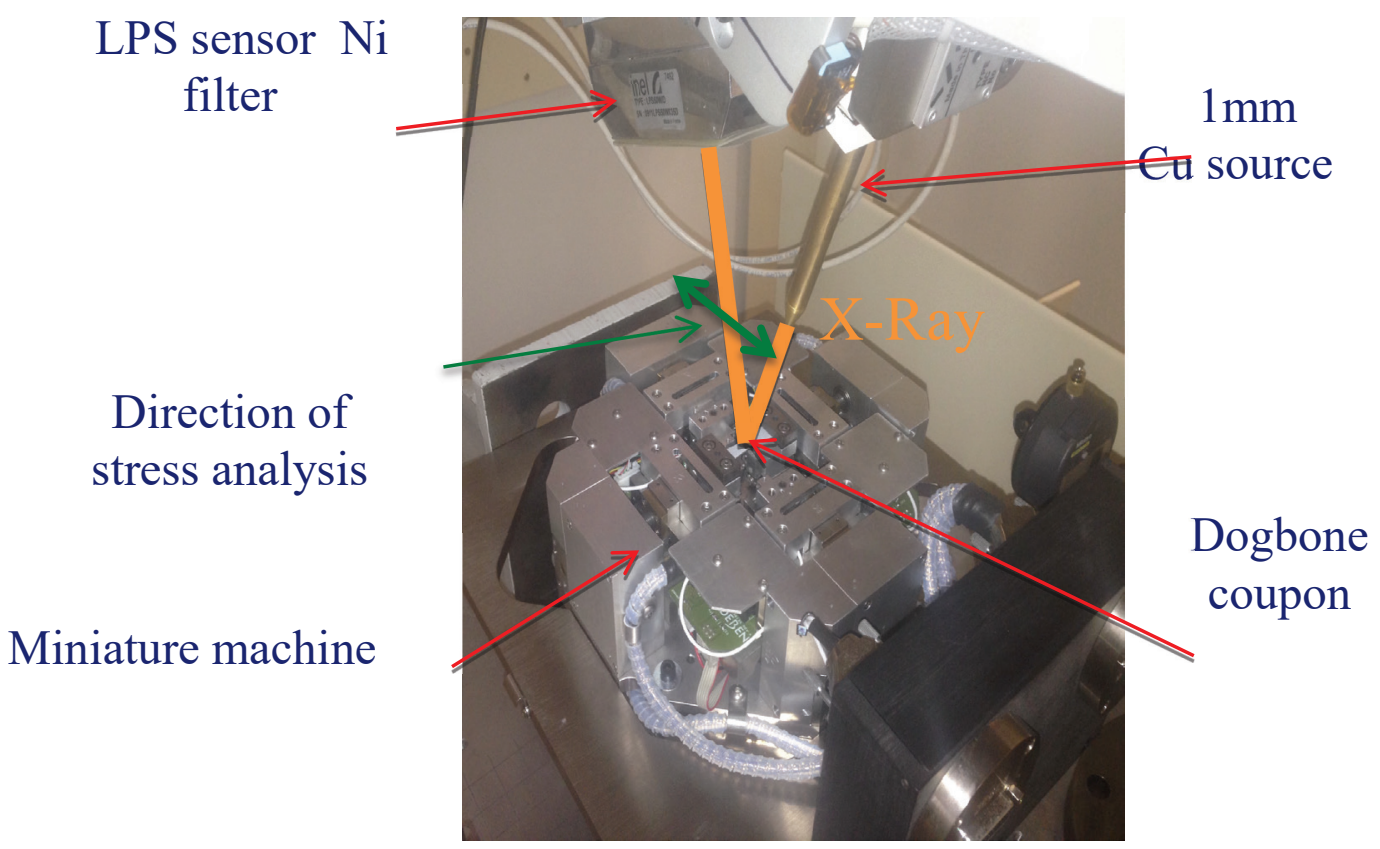

Figure 3: Dogbone Ti5553 coupon in miniature testing machine.

Parameters for XRD measurements: Cu source, 1-mm collimator, linear XRD sensor, Ni filter

The experimental procedure consists of loading the coupon at various load levels (Figure 4). The central area of specimen is left without speckle to allow for XRD measurements. Knowing transverse sections everywhere enables the mean applied stresses to be estimated, in particular in the centre of the coupon with the knowledge of measured forces. Both XRD signals and image acquisitions are carried out for each loading step. Forces and corresponding stresses vary within the range of residual stresses induced by machining $(\approx 200 \mathrm{MPa})$ or shot peening $(\approx 700 \mathrm{MPa})$, which are typical extrinsic states at the end of the manufacturing process of the studied alloy. The maximum applied stress corresponds to maximum load that can be applied by the selected testing machine. The two load levels at the middle of the experiment are longer than the other ones to assess the repeatability of the measurements, which turned out to be very good. All stress analyses via XRD are carried out in the loading direction. 


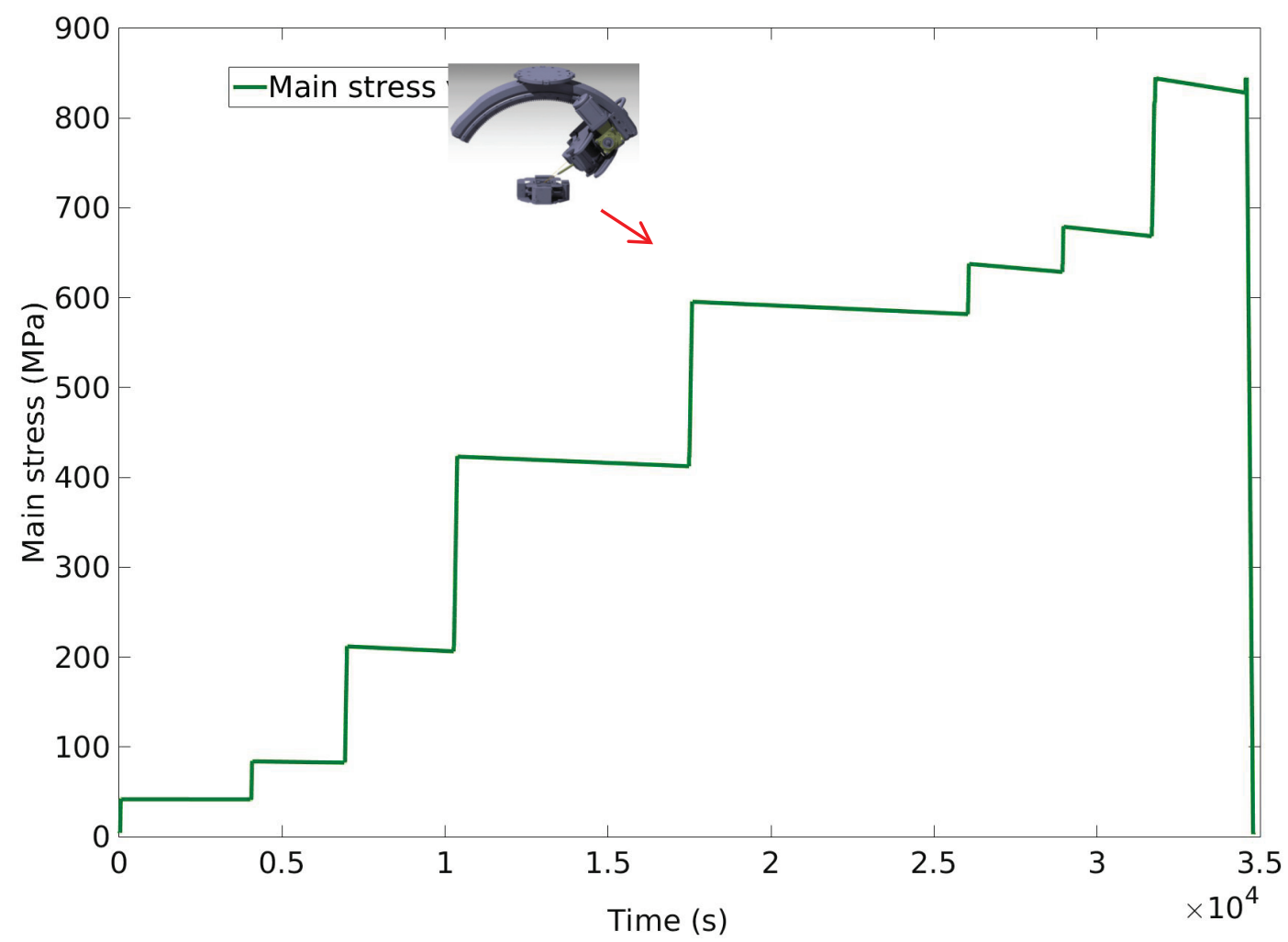

Figure 4: Load history for a Ti5553 coupon and XRD measurements

\section{Stress evaluations}

XRD diffraction peaks are registered then modelled by a Gaussian profile coupled with isotropic elasticity. It allows the peak shift to be related to stresses thanks to an integrated approach. The latter is of great interest in two-phase alloys since peaks are flat and noisy, and the determination of peak positions is not always accurate [10]. Let $f$ denote the measured diffractogram, and $g$ a reference signal (here modelled as Gaussian). An integrated approach consists of minimizing the sum of squared differences $\rho^{2}$

$$
\rho(x)=\frac{f(x)-I_{b}-D x}{\Delta I}-g\left(\frac{x-x_{0}\left(\sigma_{\phi \phi}, \sigma_{\phi 3}, \varepsilon_{0}\right)}{\sigma_{0}}\right)
$$

over the whole measurement data points $x$ and diffractograms with respect to the peak height $\Delta I$, the mean background level $I_{b}$ and its spatial drift $D$, the peak width $\sigma_{0}$, and the three mechanical unknowns $\sigma_{\phi \phi}, \sigma_{\phi 3}, \varepsilon_{0}$ via a Gauss-Newton scheme. In this formulation, the peak position $x_{0}$ is directly parameterised with the normal stress $\sigma_{\phi \phi}$, the shear stress $\sigma_{\phi 3}$, and $\varepsilon_{0}$ that is a term combining the trace of stress tensor and the reference angle [10]. The elastic and isotropic law used to model stresses once lattice strains $\varepsilon_{\phi \psi}$ are measured via XRD in the direction $\phi \psi$ reads

$$
\varepsilon_{\phi \psi}=\frac{1}{2} S_{2} \sigma_{\phi \phi} \sin ^{2} \psi+S_{1} \operatorname{tr}(\sigma)+\frac{1}{2} S_{2} \sigma_{\phi 3} \sin 2 \psi
$$

where $S_{1}$ and $S_{2}$ are the elastic parameters of the $\alpha$-phase for the considered $\{213\}$ plane family.

Figure 5 displays applied stress estimations in the analysed zone (Figure 2) for different load levels (Figure 4). The first estimation, which is referred to as digital signal correlation (DSC), uses peak registration as commonly performed in commercial codes (e.g., StressDiff). The results given by 
the integrated method, i.e., integrated digital signal correlation (IDSC), which consists of directly seeking the stresses within the peak registration procedure (i.e., the peak shift is parameterised with the unknown stresses), are also shown.

The fact that the registration residuals for DSC and IDSC are very close validates the integrated approach and also the isotropic elastic model [8]. Further, the stresses can also be estimated with the force levels measured by the load cells of the testing machine. Very similar results are obtained with the three different approaches. Part of the observed differences is due to geometric defects induced by electropolishing, which was not uniform. Figure 5 also reports the standard uncertainties of stress estimations for each load and for DSC and IDSC algorithms (green markers) and compares them to stress evaluations with the load data (black markers). The standard resolutions for both methods are similar and small $(\approx 15 \mathrm{MPa})$ compared to stress estimation levels. It is an additional validation for the registration of XRD peaks and the hypothesis of isotropic elasticity. Root mean square errors between stress estimations by DSC or IDSC and load data are higher. They include errors in evaluating the real section geometry. The mean value of these errors is $38 \mathrm{MPa}$, which remains small given all the challenges that had to be addressed.
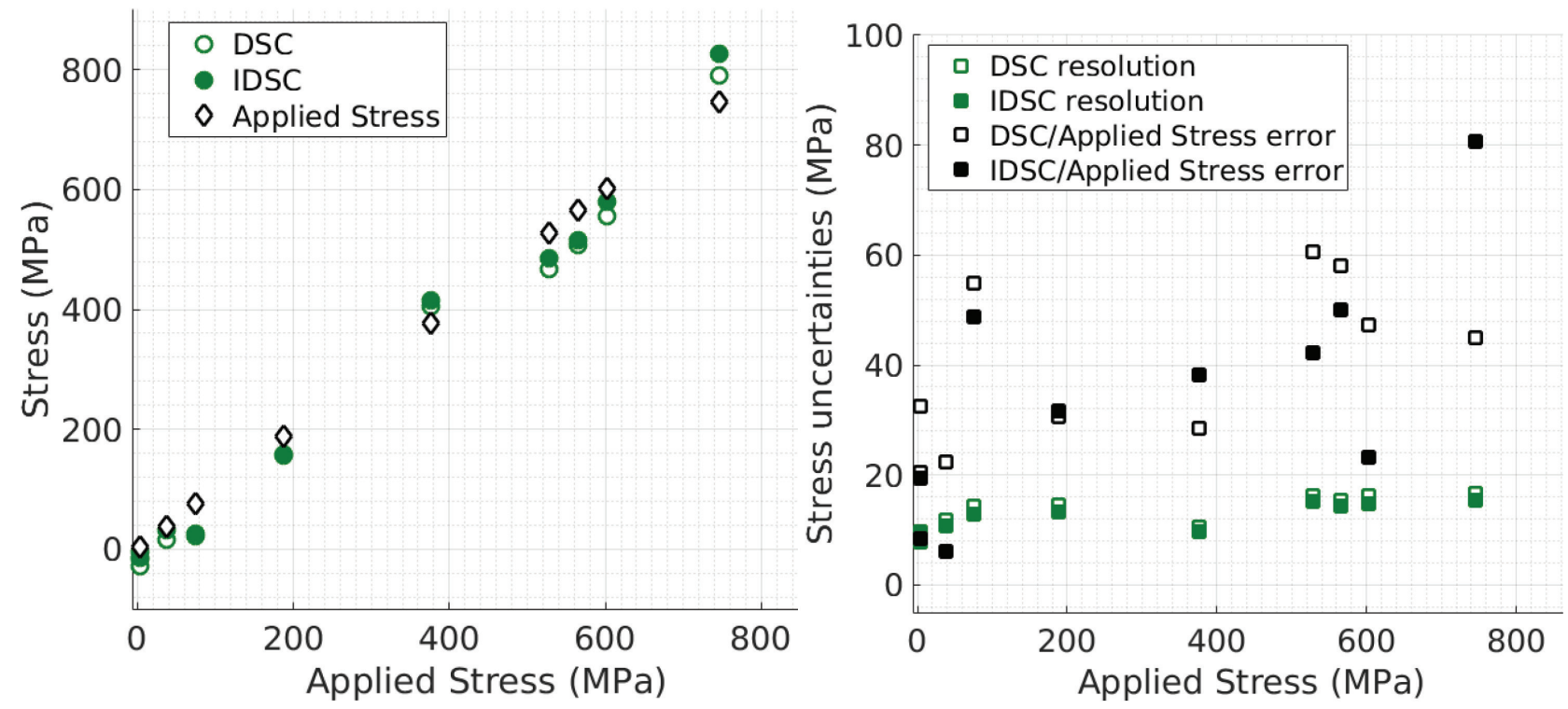

Figure 5: Estimation of stresses with different methods (left) and corresponding standard stress uncertainties (right). For each stress evaluation $13 \psi$ positions were considered [11]

\section{Conclusion}

A new procedure has been used to estimate stresses via XRD analyses using isotropic elasticity. An in-situ tensile test has been carried out to validate both experimental methodologies and an in-house integrated signal registration procedure. It has been applied to high strength Ti5553 alloy exhibiting shallow and noisy diffraction peaks.

\section{References}

[1] R. R. Boyer, R. D. Briggs, The use of $\beta$ titanium alloys in the aerospace industry, J. Mater. Eng. Perform., 14 (2005) 681-685. http://dx.doi.org/10.1361/105994905X75448

[2] E. C. Reed, J. A. Viens, The influence of surface residual stress on fatigue limit of titanium, J. Eng. Ind., 82 (1960) 76-78. http://dx.doi.org/10.1115/1.3663004

[3] A. Cox, J.P. Villain-Chastre, S. Turner, M. Jackson, The effect of finish milling on the surface 
integrity and surface microstructure in Ti-5Al-5Mo-5V-3Cr, in: Proc. $13^{\text {th }}$ World Conference on Titanium, 45, 2016. http://dx.doi.org/10.1002/9781119296126.ch45

[4] S. Roux, F. Hild, Stress intensity factor measurements from digital image correlation: postprocessing and integrated approaches, Int. J. Fract., 140 (2006) 141-157. http://dx.doi.org/10.1007/s10704-006-6631-2

[5] M.E. Fitzpatrick, A.T. Fry, P. Holdway, F.A. Kandil, J. Shackleton, L. Suominen, Determination of residual stresses by X-ray diffraction, in: Measurement Good Practice Guide No. 52, 2005

[6] A. Bhattacharjee, V.K. Varma, S.V. Kamat, A.K. Gogia, S. Bhargava, Influence of beta grain size on tensile behavior and ductile fracture toughness of titanium alloy Ti-10V-2Fe-3Al, Metall. Mat. Trans. 37A (2006) 1423-1433. http://dx.doi.org/10.1007/s11661-006-0087-x

[7] S. Fréour, D. Gloaguen, M. François, R. Guillen, E. Girard, et al. Determination of the macroscopic elastic constants of a phase embedded in a multiphase polycrystal - application to the beta-phase of Ti17 titanium based alloy, Materials Science Forum, Trans Tech Publications Inc., 404-407 (2002) 723-728

[8] J. Lu, Handbook of measurement of residual stresses, Society for Experimental Mechanics, 1996

[9] F. Lefebvre, M. François, J. Cacot, C. Hemery, P. Le-Bec, E. Baumhauer, D. Bouscaud, T. Bergey, D. Blaize, D. Gloaguen, J. L. Lebrun, A. Cosson, R. Kubler, Y. Cheynet, E. Daniel, H. Michaud, J.C. Monvoisin, P. Blanchet, P. Allain, Y. Mrini, J. M. Sprauel, P. Goudeau, P. Barbarin, C. Charles, J.M. Le Roux, W. Seiler, C. Fischer, L. Desmas, A. Ouakka, M.J. Moya, Y. Bordiec, External reference samples for residual stress analysis by X-ray diffraction, Mat. Sci. Forum 681 (2011) 215-222. http://dx.doi.org/10.4028/www.scientific.net/MSF.681.215

[10] B. Voillot, F. Hild, J.L. Lebrun, R. Billardon, Evaluation of residual stresses due to mechanical treatment of Ti5553 alloy via XRD, in: Proc. 13 ${ }^{\text {th }}$ World Conference on Titanium, 268, 2016. http://dx.doi.org/10.1002/9781119296126.ch268

[11] EN 15305:2008, Non-destructive testing - test method for residual stress analysis by X-ray diffraction, 2008 